\title{
HYBRID PROTOTYPING: PURE THEORY OR A PRACTICAL SOLUTION TO ACCELERATING PROTOTYPING TASKS?
}

\author{
Mathias, David; Hicks, Ben; Snider, Chris \\ University of Bristol
}

\begin{abstract}
Physical prototyping is critical activity in the produce development process, but the cost and time required to produce prototypes hinders it use in the design process. Hybrid prototyping through coupling LEGO and FDM printing is presented as an approach to address these issues. After establishing the separate design rules for FDM printing and LEGO, this paper created new set of rules called Design for Fabrication (DfF) for hybrid prototyping. These cover the three main considerations (Technical, Process, and Design) that the designer and process planning must include to practically implement LEGO and FDM hybrid prototyping. The DfF rules were considered in a prototype of a computer mouse. While the fabrication time was not reduced as expected, it showed that the rules could be practically implemented in a real-world example. Additional considerations were identified that are to be included in the DfF rules.

Further work is required to realise the predicted step-change reduction in fabrication time. The first approach is to leverage multiple printers to parallelise the printing. The second is to reduce fidelity while maintaining high fidelity in key regions of interest.
\end{abstract}

Keywords: LEGO, 3D printing, Physical prototyping, Early design phases, Design for X (DfX)

\section{Contact:}

Mathias, David

University of Bristol

Mechanical Engineering

United Kingdom

david.mathias@bristol.ac.uk 


\section{INTRODUCTION}

Prototyping is a crucial part of the product development process (Wall, Ulrich, \& Flowers, 1992) and it is widely accepted that increased prototyping activities lead to improved products (Menold, Jablokow, \& Simpson, 2017). In particular, the use of physical prototypes has been shown to have numerous benefits to both the designer and the outcome of the product development process. It allows the designer to explore the design space (Hess \& Summers, 2013) and learn about the design problem (Jensen, Elverum, \& Steinert, 2017) while supplementing their mental models (Viswanathan \& Linsey, 2011). Physical prototypes also give rise to unexpected phenomena (Otto \& Wood, 2001), and act as boundary objects for communication within design teams and between stakeholders (Boujut \& Blanco, 2003).

The two biggest factors hindering the use of physical prototypes in the design process are the cost and the time required to produce them (Camburn et al., 2017). To begin to address this, researchers have begun to explore the affordances of hybrid prototyping methods aiming to combine their relative affordances. This paper considers the novel approach of coupling LEGO and Filament Deposition Modelling (FDM) printing. Previous work by Mathias et al. (2019) has shown that LEGO offers flexibility and ease of use without getting fixated on producing a high fidelity prototype, while FDM methods have been developed with the specific aim of producing high fidelity form-based prototypes (Conner, Manogharan, \& Meyers, 2015). Coupling FDM printing and LEGO combines the relative affordances of both methods while mitigating their limitations. As a result, it is possible to reach levels of fidelity unachievable by LEGO alone while maintaining the flexibility and reconfigurability of a construction kit, as well as significant time and materials savings. The approach taken is a volumetric one that uses LEGO to form the internal volume of a prototype, with FDM 3D printing providing high-fidelity surfaces to attach onto the LEGO. Parallels can be drawn to CNC machining, where the LEGO is a 'rough cut' - forming the quick, approximate shape, and the FDM printing is a 'finishing pass' - creating high fidelity detail more slowly. Figure 1 illustrates how FDM and LEGO hybrid prototyping can be coupled in $2 \mathrm{D}$.

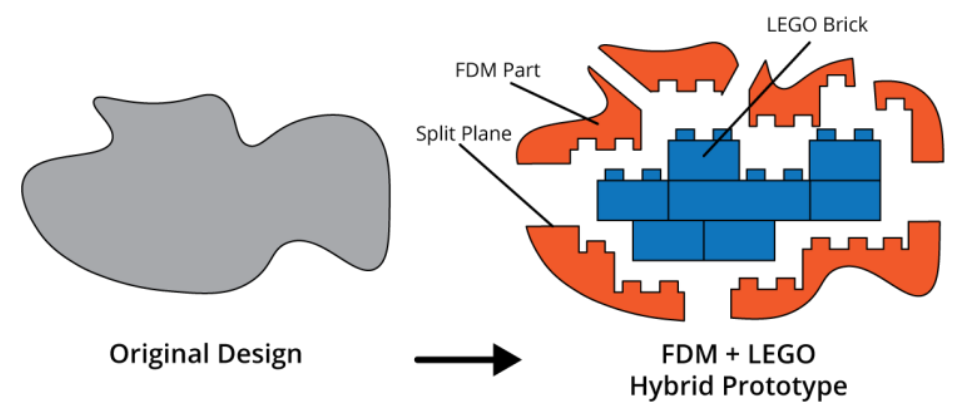

Figure 1. An illustrative 2D example of FDM and LEGO hybrid prototyping.

The affordances of this hybrid prototyping technique have been investigated through a theoretical, simulation-based study (Mathias et al., 2019), this showed that fabrication times could be reduced by $42 \%$ compared to simply FDM printing the prototype. However, this previous work does not consider the implementation of the approach - as a new physical prototyping technique it needs to be both feasible and practical for it to be usefully employed in the design process. Correspondingly, the motivation for this paper concerns the physical implementation of the LEGO and FDM hybrid prototyping. As the benefits have been shown, the ability to produce the prototypes would determine whether it represents solely a theoretical proposition or whether it can be a practical solution to accelerating prototyping tasks.

The contribution of the paper is to demonstrate and evaluate the viability of hybrid prototyping through the development and integration of a set of design rules for design, fabrication and assembly. The design considerations include attention to overall form or minimum feature size. The fabrication and assembly perspectives concern process planning for fabrication, and in particular, how to segment and then print the high-fidelity parts; and, how the LEGO bricks should be assembled together to create a prototype of appropriate structural integrity, that is a prototype that is at least self-supporting. The paper begins with a summary of physical prototyping and the need for hybrid prototyping, before considering existing design rules for additive manufacture and LEGO assembly principles. The design for fabrication and assembly (DfFA) rules for hybrid prototyping with FDM printing and LEGO are 
then presented and illustrated using a case study. The paper concludes with a discussion and reflection on the considered approach, with future work posited.

\section{BACKGROUND}

While physical prototyping can be used at almost any stage of the product development process, in this paper we are considering the early stages of the design process that use form prototypes, as opposed to function ones (Hallgrimsson, 2012). These types of prototypes are frequently used as learning and communication tools (Ulrich \& Eppinger, 2016) that allow designers to understand and explore their designs and to explain and discuss ideas with stakeholders or clients.

Physical prototyping techniques that are commonly used in these early stages include: construction kits, foam and cardboard modelling, clay modelling, and low cost FDM printing. Mathias et al. (2018) consider these physical prototyping techniques on a spectrum of fidelity and reconfigurability, as shown in Figure 2. For designers, there is a decision that has to be made that trades-off quality and time when choosing a suitable technique from this spectrum (Sass \& Oxman, 2006).

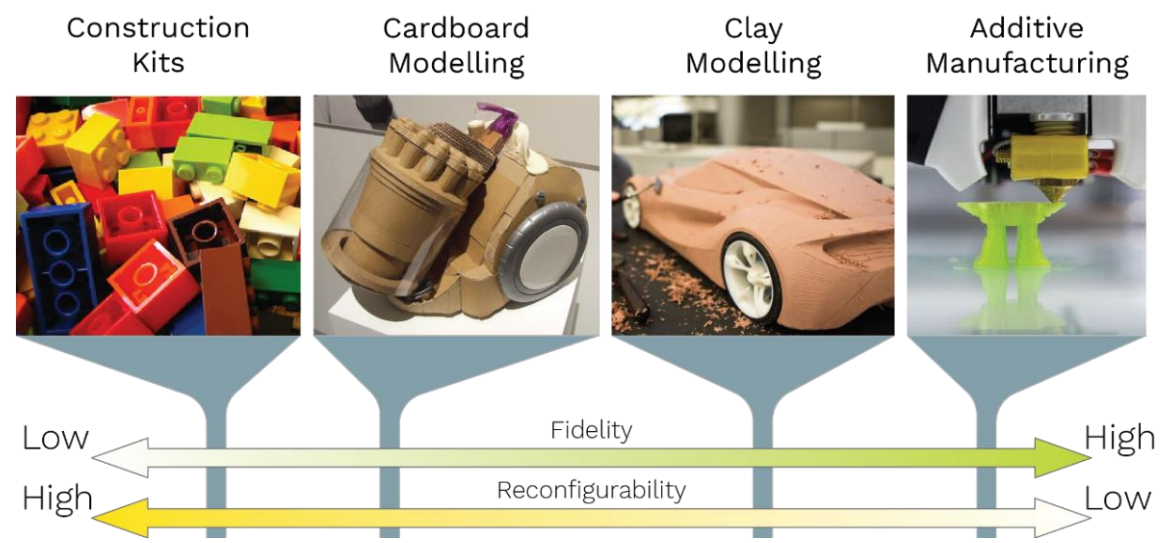

Figure 2. Common physical prototyping techniques occupying a spectrum of fidelity and reconfigurability.

As shown by this spectrum, there is no single technique that affords reconfigurable, high fidelity prototypes that can be quickly fabricated.

There have been considerable research efforts to improve prototyping - these can be broken down into two main categories: process and technological improvements. Process improvements have tended to focus on heuristic frameworks that help designers to manage their prototyping endeavours (Menold $e t$ al., 2017) and usually prescribe what techniques to use and when to apply them in the design process. Despite these frameworks, the largest barriers to using physical prototypes in the product development process are the cost and time required to produce them (Otto \& Wood, 2001). Proposed technological improvements to overcome these issues include: the use or reuse of existing products and components (Camburn et al., 2017); accelerating digital fabrication through wire printing and laser cutting by sacrificing fidelity (Beyer, Gurevich, Mueller, Chen, \& Baudisch, 2015). To increase prototype flexibility and reusability, approaches such as editable physical models have been proposed (Lennings, Broek, Horváth, Sleijffers, \& de Smit, 2000); and methods for adapting LEGO to be more suited to higher fidelity prototyping have been presented (Boa, Mathias, \& Hicks, 2017).

Hybrid prototyping attempts to overcome the issues of fabrication time and production cost by coupling two different prototyping techniques so that their complementary affordances can be combined, and their respective limitations mitigated. Two reported uses of hybrid prototyping in industry include:

- Using a mix of cardboard and plastic parts in the development of new vacuum cleaners (James Dyson Foundation, 2010). The plastic parts are designs fixed from previous iterations, while the cardboard parts are still being designed allowing cheap modification and editability.

- Using sculpting clay over a wooden frame to generate the body shape of cars in the automotive industry (Singh, 2006). The structural frame gives the rough shape and support for the prototype while the clay allows the designers to create detailed, complex curves.

In literature, there has been previous work on combining LEGO and FDM printing (Mueller, Mohr, Guenther, Frohnhofen, \& Baudisch, 2014). This work only focused on low-fidelity/high-speed and did 
not consider a more general and practical physical implementation. However, it did show the potential of coupling LEGO and FDM printing and that it is a way to address the issues in physical prototyping, but to date it is a theoretical proposition not a practical solution.

\section{DESIGN RULES FOR PROTOTYPING WITH FDM AND LEGO}

While current approaches to hybrid prototyping are rather ad-hoc and have been shown to offer benefits, there is value in providing a more consistent framework and approach to maximise these benefits. This includes ensuring optimal time/cost/quality when producing prototypes. It also provides potential for computational offloading and automation, such as the techniques used by Mueller et al. to automatically generate the LEGO brick layout.

To develop new design rules for hybrid prototyping with LEGO and FDM printing, the existing design rules and considerations for each prototyping method are first reviewed. Correspondingly, the following sections summarise relevant literature for design for additive manufacture (DfAM) and LEGO assembly. The contributions of this paper are tied to LEGO and FDM printing; while significant similarities with other methods exist, these would need to be designed/studied specifically.

\subsection{Design for additive manufacture}

Design for additive manufacture is dependent on the specific additive manufacture (AM) process that is used - with each process having different rules and considerations. However, as the hybrid prototyping technique in this paper is focussing on FDM printing, the DfAM review will only consider those relevant to FDM printing. There are two areas in DfAM that the designer must understand: process considerations and geometric considerations (Goguelin, Flynn, \& Dhokia, 2016).

The process considerations include the capability of the printers (size, accuracy, speed), material properties (strength, temperature, part anisotropy), and the print settings (layer height, infill percentage, wall thickness, support material etc). While the geometric considerations focus on the artefact being designed. Many of these variables are fixed by the use of desktop FDM printers - particularly with regard to material properties (typically limited to PLA or ABS) and capability of the printers. A typical desktop FDM printer (Ultimaker B. V., 2018) has an approximate build volume of $200 \times 200 \times 200 \mathrm{~mm}$, with X and $\mathrm{Y}$ resolutions, and $\mathrm{Z}$ resolutions of 12.5 microns and 20 microns respectively.

The print settings used impact the print time, part strength and quality but are not affected by the geometry of the part. For geometric considerations, there are guidelines on recommended values for particular geometric features in the design of FDM printed parts, as shown in Table 1.

Table 1. Guide values for geometric features on FDM printed parts (Redwood et al., 2017).

\begin{tabular}{|l|l|l|l|l|l|}
\hline Feature & Guide & Limit & Feature & Guide & Limit \\
\hline Wall Thickness & $0.8 \mathrm{~mm}$ & Min & Clearance & $0.5 \mathrm{~mm}$ & Min \\
\hline Overhangs & $45^{\circ}$ & Min & Feature Size & $2.0 \mathrm{~mm}$ & Min \\
\hline Engraved Details & $0.6 \times 2 \mathrm{~mm}$ & Min & Pins & $\varnothing 3.0 \mathrm{~mm}$ & Min \\
\hline Bridges & $10 \mathrm{~mm}$ & Max & Unsupported Edges & $3.0 \mathrm{~mm}$ & Max \\
\hline Holes & $\varnothing 2.0 \mathrm{~mm}$ & Min & & & \\
\hline
\end{tabular}

Two aspects that bridge between process and geometry considerations are the print orientation and supported overhangs. While these aspects are dictated by how the printing process is setup, they need to be taken into account during the design process to ensure the printed parts meet requirements. Printed parts exhibit anisotropic behaviour under loading due to their layered construction, as a result it is important, where possible, to ensure that parts are loaded parallel to layers to prevent delamination. While support material can be added to create overhangs and bridges, it takes longer to print and often results in an undesirable surface finish, hence overhangs need to be considered during the design process. Some geometric modifications can be applied to reduce the need for support material, such as adding chamfers under unsupported edges or ensuring circular profiles are changed to teardrop ones to prevent drooping at the top of the circle.

\subsection{Design for LEGO assembly}

Although LEGO was originally designed as a children's toy, it has seen increased use as a tool for education and design - evidenced by the LEGO Group's creation of the Architecture Studio (The 
LEGO Group, 2013), allowing architects to quickly explore new building designs. As a result, there are established best practices that ensure that resulting models, whether a design output or toy kit, can be assembled and are strong enough to hold together.

Before describing different assembly techniques, there are fundamental aspects of LEGO that need to be considered when designing parts to be made from the construction kit. Firstly, LEGO bricks are discrete and can only be constructed at a fixed scale of $8 \mathrm{~mm}$, in fixed, orthogonal planes. This means that objects can only have dimensions that are integer numbers of bricks and that complex curves can only be approximated with a 'pixelated' appearance. While there are more advanced techniques that mitigate this limitation, they are more challenging to implement and impact significantly on the reconfigurability of models and require substantial design effort (Enjary, 2007). Secondly, the size, and finite library, of LEGO bricks (the smallest being $8 \times 8 \times 3.2 \mathrm{~mm}$ ) means that there is a fixed lower bound of an object's size. On the other hand, while very large LEGO assemblies are possible, they are frequently too complex and costly to be viable outside LEGOLAND theme parks - giving rise to an upper bound for reasonably sized LEGO models.

Gower et al. (1998) identify some general construction rules for creating stable LEGO structures:

- The use of large bricks.

- Alternating directionality of bricks in consecutive layers.

- High proportion of overlap of each brick's area (above and below) by other bricks.

- High proportion of each brick's vertical boundary is covered (above and below) by other bricks.

These rules do not include any design considerations that would affect the geometry or possible designs of the assembled model - they are purely focussed on creating assemblies that have structural integrity. Luo et al. (Luo et al., 2015) established a forced based approach to optimising LEGO assemblies, through experimentation they found that the separation force between two bricks was $0.703 \mathrm{~N}$ per stud. This is a conservative value, measured through worst case loading. By creating LEGO assemblies that do not exceed this value, there is sufficient structural integrity to support their own-weight. Applying this to the joint between two 2x2 LEGO bricks, the join can support up to $286 \mathrm{~g}$ (or $2422 \times 2$ bricks) before failing.

The Architecture Studio (The LEGO Group, 2013) offers higher-level assembly techniques that not only consider the strength of the design, but also how to overcome some of the limitations of LEGO as well as add small details to the architectural designs. These techniques are found in Table 2.

Table 2. A table showing the LEGO architecture studio assembly techniques.

\begin{tabular}{|l|l|}
\hline Locking & Placing a brick over the join of two other bricks to increase strength \\
\hline Sideways Building & Use bricks with studs on their sides to build sideways \\
\hline Size-Scaling & Build at small scale to show full scale objects \\
\hline Details & Select textured/smaller bricks to show surface details \\
\hline Alternative Uses & Use bricks in novel/different ways to create your designs \\
\hline Building in Sections & Build smaller modules and bring them together later \\
\hline
\end{tabular}

Other than the Locking technique, all these guidelines are design considerations that the designer can use to build prototypes that are representative of their concepts.

\section{METHODOLOGY}

This section covers the development of the design rules for hybrid prototyping and illustrates their application to the prototyping of a computer mouse.

Figure 1 shows a 2D example of a LEGO and FDM hybrid prototype. The LEGO assembly forms the internal volume, while a hollow outside surface is FDM printed. This surface has to be segmented into parts so that it can be assembled or modified. The separation of these parts occurs at split planes these do not have to be planar and could be more complex surfaces. Finally, the level of fidelity of a hybrid prototype determines how much of the outer form is FDM printed versus how much is made from LEGO. In the case study considered in this paper, the level of fidelity is not reduced, and the entirety of the outer form is FDM printed.

\subsection{Hybrid prototyping design rules}

From the existing design rules for FDM printing and LEGO, it is possible to separate the rules into two groups: ones that are technical capabilities/limitations of the technique, and ones that the designer 
must consider when creating the design's geometry. In DfAM, these are called process and geometric considerations respectively. However, in coupling LEGO and FDM printing as a hybrid prototyping technique, a finer level of granularity is required in order to accommodate the rules that influence the process plan and ones that require input from the designer. As a result, we are considering three groups of design rules: Technical Constraints, Design Considerations, and Process Considerations. Figure 3 shows the relationship between the three groups of design rules, the design and planning (blue), and the output hybrid prototype (green). The process and design considerations need the designer's input (red), while the technical considerations are fixed by the use and coupling of FDM printing and LEGO (orange). The technical considerations and designer's process decisions are combined in the process planning that generates the LEGO assembly and segmented FDM printed parts from the prototype geometry.

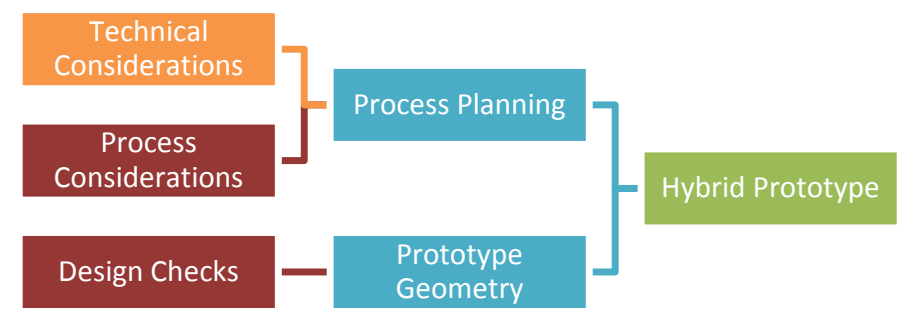

Figure 3. The relationship between the design rules and the hybrid prototype.

The following sections cover the rules established for each of the three groups for hybrid prototyping. While reduction in fabrication time is cited as one of the key benefits of coupling LEGO and FDM printing (Mathias et al., 2019), if the prototype cannot be made or is not self-supporting then the time reduction is irrelevant.

\subsubsection{Technical considerations}

The technical constraints are fixed and cannot be impacted by the actions of the designer. These constraints inform the decisions and calculations that in turn drive the process planning that generates the LEGO assembly and FDM printed geometry.

- Assembly/Disassembly - The prototypes must be able to be put together and taken apart. As a result, the LEGO pieces must be accessible via the split planes that separate the FDM printed outside surface.

- $\quad$ Structural integrity - The hybrid prototype must be strong enough to be handled and support its own weight. This should be achieved by following the LEGO structure assembly rules described in Section 3.2. The process planning must layout the bricks to reduce the loading placed on any single stud to the acceptable bounds (0.703 N (Luo et al., 2015)).

- Composition of LEGO set - The library of available brick sizes and their number needs to be specified in advance for the process planning to ensure that it does not generate prototypes that cannot be built due to insufficient available bricks.

- Number and size of the FDM printers - The generated FDM printed parts must be small enough to be produced by the available printers. If more than one printer is available, there are opportunities to parallelise the printing and further reduce fabrication time as the printing time can be shared across multiple printers.

Assembly and structural integrity do not have fixed values that constrain the output (unlike the type/number of LEGO bricks available or size of the printers) but rather represent minimum bounds that need to be met during the process planning stage. As a result, there needs to be optimisation performed on the layout of the LEGO bricks and location of the segmentation slices of the FDM printed parts. In order to achieve this, parameters for cost minimisation algorithms (similar methods employed by Gower et al. (1998)) need to be determined.

\subsubsection{Design considerations \& checks}

The design checks are aspects that the designer must take into account when they are specifying the form, shape and geometry of the prototype. If the design fails these checks then it is not suitable for FDM and LEGO hybrid prototyping. 
- Overall size - The design must fit between certain overall dimensional bounds that ensure that hybrid prototyping is a suitable method to embody the prototype. If it is too small (around LEGO brick size) then the design cannot accommodate any LEGO and will be entirely FDM printed. Conversely, if the design is too big (larger than desktop sized), then the high number of bricks required and resulting slow fabrication time makes other prototyping techniques more suited.

- Minimum dimension/thickness - Similar to the overall size, the design cannot have a dimension or thickness smaller than a LEGO brick (i.e. a thin plate or thin-walled box). The smallest LEGO brick is $8 \times 8 \times 4 \mathrm{~mm}$ (including the stud) and so allowing for connection between the brick and FDM part, the dimensions of the prototype must be larger than $10 \mathrm{~mm}$. However slender regions can exist so long as other parts of the design are large enough to be constructed out of LEGO.

- Feature size - As the outside surface of the prototype is FDM printed, any features must conform with the DfAM recommended values described in Table 1. Overhang angle and unsupported edge length can be excluded as the FDM parts will be re-orientated for printing.

\subsubsection{Process considerations}

The process considerations are designer decisions that do not affect the design geometry and dictate what variables the process planning should use to create the hybrid prototype. These are akin to the print settings in slicing software for FDM printing.

- Size/number of segmented FDM parts - Choosing between fewer, larger parts (with fewer splits) or a greater number of smaller parts allows the designer to control the level of modularity and flexibility the prototype has. Furthermore, if more than one printer is available then a greater number of parts will mean that the overall fabrication time can be reduced.

- Location of split planes - As the outer surface has to be segmented, the split planes may intersect a critical feature. This would allow the designer to choose which particular feature or geometry get preserved. For example, ensuring that a button on an interface remains complete. However, this would not override the technical consideration of the requirement to be able to assemble/dissemble the prototype.

- Level of fidelity - Extending the theoretical findings of Mathias et al. (2019) it can be contended that the fabrication time can be further reduced by only printing the areas of required high fidelity. This consideration would allow designers to select the regions of interest in their design requiring higher fidelity leaving the remaining regions to be approximated by LEGO.

- LEGO usage - Another way to reduce fabrication time and LEGO brick usage, is to generate a hollow LEGO assembly. However, this would impact the strength and weight of the prototype. The designer must consider what they want to achieve with the prototype.

- FDM printer settings - Print settings have a large impact on the output from the printer, particularly with regard to a part's strength (Goudswaard, Hicks, \& Nassehi, 2018). However, in hybrid prototyping the strength of the prototypes is limited by the inter-part connection therefore the standard printed, regardless of orientation can be considered to be sufficiently strong. The settings for print speed (which will affect the fabrication time) are printer and filament dependent and need to be determined for specific printers.

\section{ILLUSTRATIVE CASE \& DISCUSSION}

To illustrate the application and implementation of the DfFA rules, the fabrication of a prototype of a computer mouse is considered. Figure 4 shows a rendering of the computer mouse and the partially constructed hybrid prototype. The internal LEGO volume is clearly visible with the FDM printed parts assembled to the underside and upper side of the prototype. The LEGO internal volume calculations were performed automatically, but the process planning for the location of the split planes, layout of the LEGO and part orientation for FDM printing were done manually. The number of resulting FDM printed parts was considered (i.e. to lie between, at most, 110 very small parts - one for every exposed study/hole - and two parts) but not optimised. Similarly, the locations of split planes were chosen to ensure access to the LEGO bricks for assembly/disassembly. The structural integrity was sufficiently strong enough to hold together when handled. 

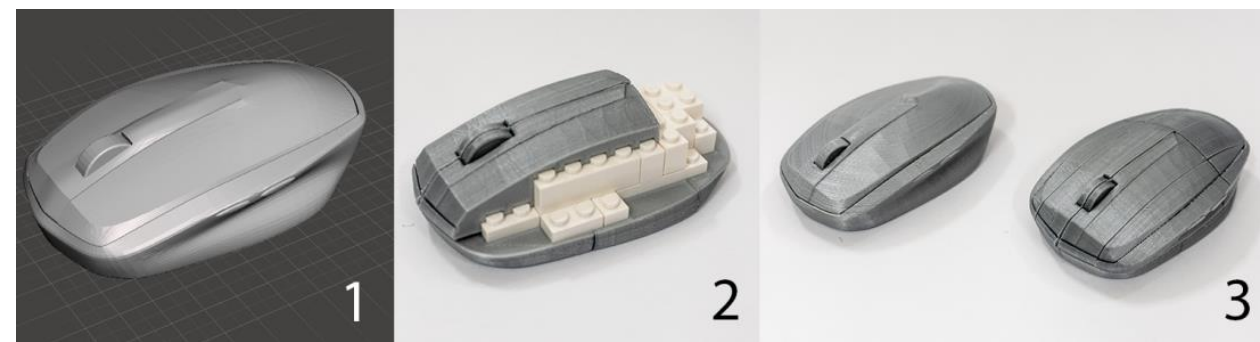

Figure 4: The digital representation of the computer mouse design (1) and the partially constructed hybrid prototype (2). Fully FDM printed prototype (3 left) and LEGO and FDM hybrid prototype (3 right).

Figure 4 shows a fully FDM printed prototype next to the LEGO and FDM hybrid prototype. The fully printed version took 246 minutes to print, while the hybrid prototype took 393 minutes to print (12 parts) and another 11 mins to assembly together with the 24 LEGO bricks. In both cases, identical print settings were used on a single printer.

\subsection{Discussion}

The illustrative case shows that the DfFA rules make hybrid prototyping feasible and practical. From enacting the DfFA rules manually, several observations can be drawn regarding the additional considerations that would need to be included for hybrid process planning. These are:

- Orientation/support material - Once segmented, the process planning must consider and optimise the orientation of the FDM parts to minimise print time and requirement for support material.

- Location of the split planes - Some of the split planes ran through holes/studs on the FDM parts, causing the LEGO/FDM interface to be less secure and harder to assemble. Where possible, this should be avoided in order to keep holes/studs intact.

- Connection between FDM parts - Although flat surfaces were used as split planes, positive connection between FDM parts would be useful, rather than relying on the LEGO interface.

- Need for instructions - Despite the relatively small number of bricks (24) and FDM parts (12), the computer mouse (Figure 4) was not simple to assemble. The addition of instructions would expedite the assembly process and result in fewer mistakes.

However, in this illustrative case, the overall fabrication time for the hybrid-based prototype was greater than for a single piece prototype created via FDM printing. This is interesting from a technical point of view and would need addressing to achieve the possible step change in reduction in fabrication time suggested by Mathias et al. (2019). This could be realised through two complementary approaches; the parallelisation of the printing process through the use of multiple printers; and varying the level of fidelity of surface detail (resolution) to match the needs of the designer by only printing regions that need to be high fidelity. However, these are out of scope of this paper.

It follows that for DfFA to be practically possible and to realise the significant reduction in prototype fabrication times, means to optimise the number and size of FDM printed parts with respect to print time must be created, and is a crucial to make hybrid prototyping a viable and beneficial approach.

\subsection{Future work}

The work reported in this paper forms part of a larger body of research aimed at creating an automated tool for the task of generating and fabricating hybrid prototypes. The key components of such an automated tool include:

- Design and geometry checks to ensure suitability for hybrid prototyping prior to process planning.

- Capturing fidelity requirements or intent to preserve feature geometries and reduce detail and hence print requirements.

- Generation of FDM parts to be printed, with optimisation for the distribution of parts and print times over the available printers.

- Optimisation and brick count reduction of LEGO for assembly time and structural integrity.

- Automated generation of assembly instructions for the LEGO bricks and FDM parts. 
With respect to this overarching aim and the illustrative case study, it is evident that further work is required to develop the DfFA rules into a prototyping approach that realises the benefits described by Mathias et al. (2019).

An investigation into the size of prototypes and resulting fabrication times is required to verify whether segmenting and printing multiple parts consistently results in slower print times. This would impact the necessity of parallelisation or adapting fidelity. From this, understanding the factors and performance indicators for parallelisation can be established so that optimisation algorithms can be developed that balance fabrication time, FDM part size and number of printers. In order to be able to adapt prototype fidelity, relative measures for the required fidelity of a prototype should be established as well as methods for capturing the designer's desired region of interests or intent.

\section{CONCLUSION}

After establishing the separate design rules for FDM printing and LEGO, this paper created new set of rules called Design for Fabrication and assembly (DfFA) for hybrid prototyping. These cover the three main considerations (Technical, Process, and Design) that the designer and process planning must include to feasibly and practically implement LEGO and FDM hybrid prototyping. The DfFA rules were considered in an illustrative case study of a prototype of a computer mouse (see Figure 4). While the fabrication time was not reduced as expected by Mathias et al. (2019), it showed that the rules could be practically implemented in a real-world example. From this, additional considerations were identified that are to be included in the DfFA rules.

\section{ACKNOWLEDGEMENTS}

The work reported in this paper has been undertaken as part of the ProtoTwin project (Improving the product development process through integrated revision control and twinning of digital-physical models during prototyping). The work was conducted at the University of Bristol in the Design and Manufacturing Futures Lab (http://www.dmf-lab.co.uk) and is funded by the Engineering and Physical Sciences Research Council (EPSRC), Grant reference EP/R032696/1.

\section{REFERENCES}

Beyer, D., Gurevich, S., Mueller, S., Chen, H.-T. and Baudisch, P. (2015), "Platener: Low-Fidelity Fabrication of 3D Objects by Substituting 3D Print with Laser-Cut Plates", In Proceedings of the ACM CHI'15 Conference on Human Factors in Computing Systems, Seoul Korea, Vol. 1, pp. 1799-1806. http://doi.org/10.1145/2702123.2702225

Boa, D., Mathias, D. and Hicks, B. (2017), "Evolving lego: Prototyping requirements for a customizable construction kit", In Proceeding of 21st International Conference on Engineering Design, ICED 2017, The Design Society, Vancouver, Canada, Vol. 4, pp. 297-306.

Boujut, J.-F. and Blanco, E. (2003), "Intermediary Objects as a mean to foster Co-operation", Engineering Design Computer Supported Cooperative Work, (1998), pp. 205-219.

Camburn, B., Viswanathan, V., Linsey, J., Anderson, D., Jensen, D., Crawford, R., ... Wood, K. (2017), "Design prototyping methods: state of the art in strategies, techniques, and guidelines", Design Science, Vol. 3 No. 13. https://doi.org/10.1017/dsj.2017.10

Conner, B. P., Manogharan, G. P. and Meyers, K. L. (2015), "An assessment of implementation of entry-level 3D printers from the perspective of small businesses", Rapid Prototyping Journal, Vol. 21 No. 5, pp. 582597. https://doi.org/10.1108/RPJ-09-2014-0132

Enjary, D. (2007), “The Unofficial LEGO Advanced Building Techniques Guide”, Retrieved November 8, 2018, from https://joncraton.org/media/files/UnofficialLEGOAdvancedBuildingTechniquesGuide.pdf

Goguelin, S., Flynn, J. M. and Dhokia, V. (2016), "A bottom-up design framework for CAD tools to support design for additive manufacturing. Smart Innovation”, Systems and Technologies, Vol. 52, pp. 411-421. https://doi.org/10.1007/978-3-319-32098-4_35

Goudswaard, M., Hicks, B. and Nassehi, A. (2018), "Towards the Democratisation of Design: Exploration of Variability in the Process of Filament Deposition Modelling in Desktop Additive Manufacture", Transdisciplinary Engineering Methods for Social Innovation of Industry 4.0, (October). https://doi.org/10.3233/978-1-61499-898-3-125

Gower, R., Heydtmann, A. and Petersen, H. (1998), "Lego: Automated Model Construction”, European Study Group with Industry, pp. 81-94.

Hallgrimsson, B. (2012), Prototyping and Modelmaking for Product Design. Laurence King Publishing, London. 
Hess, T. and Summers, J. D. (2013), “Case study: Evidence of prototyping roles in conceptual design”, In Proceedings of the International Conference on Engineering Design, ICED13, The Design Society, Seoul Korea, Vol. 1, pp. 249-258.

James Dyson Foundation. (2010), Engineering Box - Teacher's Pack. James Dyson Foundation. Retrieved fromhttp://www.jamesdysonfoundation.co.uk/resources/engineering-box-teachers-pack/

Jensen, M., Elverum, C. and Steinert, M. (2017), "Eliciting unknown unknowns with prototypes: Introducing prototrials and prototrial-driven cultures", Design Studies, Vol. 49, pp. 1-31. https://doi.org/10.1016/j.destud.2016.12.002

Lennings, A. F., Broek, J. J., Horváth, I., Sleijffers, W. and de Smit, A. (2000), "Editable Physical Models for Conceptual Design", In Proceedings of the TMCE2000 Symposium. Delft, Netherlands.

Luo, S.-J., Yue, Y., Huang, C.-K., Chung, Y.-H., Imai, S., Nishita, T., ... Chen, B. (2015), "Legolization: Optimizing LEGO Designs", ACM Transactions on Graphics, Vol. 34 No. 6, pp. 1-12. https://doi.org/10.1145/2816795.2818091

Mathias, D., Hicks, B., Snider, C. and Ranscombe, C. (2018), "Characterizing the Affordances and Limitations of Common Prototyping Techniques to Support the Early Stages of Product Development”, In International Design Conference - Design 2018, Dubrovnik, Croatia, pp.1257-1268.

Mathias, D., Hicks, B., Snider, C. and Ranscombe, C. (2019), “Accelerating Product Prototyping Through Hybrid Prototyping Methods: Investigating and Characterising the Affordances of Coupling of FDM Printing and LEGO”. Submitted to Design Studies, Vol. 62, pp. 68-99. https://doi.org/10.1016/j.destud.2019.04.003

Menold, J., Jablokow, K. W. and Simpson, T. W. (2017), "Prototype for X (PFX): A holistic framework for structuring prototyping methods to support engineering design. Design Studies, Vol. 50, pp. 70-112. https://doi.org/10.1016/j.destud.2017.03.001

Mueller, S., Mohr, T., Guenther, K., Frohnhofen, J. and Baudisch, P. (2014), "faBrickation : Fast 3D Printing of Functional Objects by Integrating Construction Kit Building Blocks”, In Proceedings of the SIGCHI Conference on Human Factors in Computing Systems, Toronto, Canada, pp. 3827-3834. https://doi.org/10.1145/2556288.2557005

Otto, K. and Wood, K. (2001), Product Design: Techniques in Reverse Engineering and New Product Development (1st ed.), Prentice-Hall, New Jersey.

Redwood, B., Schoffer, F. and Garret, B. (2017), The 3D Printing Handbook. 3D Hubs B.V., Amsterdam.

Sass, L. and Oxman, R. (2006), "Materializing design: The implications of rapid prototyping in digital design", Design Studies, Vol. 27 No. 3, pp. 325-355. https://doi.org/10.1016/j.destud.2005.11.009

Singh, K. (2006), "Industrial motivation for interactive shape modeling: a case study in conceptual automotive design”, ACM SIGGRAPH 2006 Courses, pp. 3-9. https://doi.org/10.1145/1185657.1185671

The LEGO Group. (2013), LEGO Architecture Studio. The LEGO Group.

Ulrich, K. T. and Eppinger, S. D. (2016), Product Design and Development (6th ed.), McGraw-Hill Education, New York.

Ultimaker B. V. (2018), Ultimaker 3. Retrieved from https://ultimaker.com/en/products/ultimaker-3

Viswanathan, V. and Linsey, J. (2011), "Understanding physical models in design cognition: A triangulation of qualitative and laboratory studies", Proceedings - Frontiers in Education Conference, FIE, pp. 3-8. https://doi.org/10.1109/FIE.2011.6142848

Wall, M. B., Ulrich, K. T. and Flowers, W. C. (1992), "Evaluating prototyping technologies for product design", Research in Engineering Design, Vol. 3 No. 3, pp. 163-177. https://doi.org/10.1007/BF01580518 